\title{
Testing if Habitat and Movements Determine the Threats and Trends for Common Birds in Europe
}

\author{
Alfonso Balmori ${ }^{1^{*}}$ and Alfonso Balmori-de la Puente ${ }^{2}$ \\ ${ }^{1}$ Consejería de Medio Ambiente, Junta de Castilla y León, Valladolid, Spain \\ ${ }^{2} \mathrm{C} /$ Navarra, 1- $5^{\circ}$ B 47007 Valladolid, Spain
}

\begin{abstract}
There is a growing concern about the biodiversity crisis the planet is suffering due to increasingly widespread human activity. A key question is whether only the species with threatened status should be managed by policy makers or whether also common species deserve consideration. This study is devoted for common birds, because for the threatened species the decline factors are much better known. As a result of a thorough search, a comparison at the continental scale focusing on European common birds and analysing their main threats and trends depending on their habitats and movements (migratory or sedentary character) has been done. This is therefore a theoretical study that tries to investigate the relationships between these four variables that condition in an important way the state of their populations. The different categorical variables (habitats, movements, threats and trends) were checked together in order to find some relationships between them, using log-linear analysis or contingency tables depending on the number of variables considered. We can conclude that "Threats" are different between both "Habitats" and "Movements" of common birds, which also interact between them. The trends in European common birds are slightly influenced by the habitat they occupy which is at the same time associated with their movements. Studies with this approach in different areas could be valuable to analyse common patterns that could guide conservation efforts and action plans in specific directions and increase their efficiency with less expenditure of resources.
\end{abstract}

Keywords

Action plans, Common species, Farmland birds, Forest birds, Habitat management

\section{Introduction}

There is a unanimous scientific opinion that nature is currently undergoing a general biodiversity crisis [1-3]. Widespread human activity results in the decline of many species and the increase in a few that thrive in disturbed environments $[4,5]$. Moreover, the few species which succeed and increase their numbers in the changing areas do not necessarily mean a biodiversity gain: For example, the increase in freshwater birds due to water eutrophication, when nutrient enrichment in some dunes and marshes facilitate the spread of shrubs, with a consequent enrichment of bird species [4].

In the face of the global biodiversity crisis, the European Union has adopted the target of halting the loss of biodiversity by 2010 . The sustainable use of resources is a central pillar of the Convention on Biological Diversity (CBD) [5].

Usually, efforts have been focused on most endangered species and for this reason, the different drivers, pressures and threats for a small number of rare or threatened species, with unfavourable population situations and extremely declining trends, are generally well known [6]. Conservation plans developed for individual species can serve to protect others as an "umbrella", but this is not always the case and usually less attention has been paid to the problem of depletion of more common and widespread breeding birds [7,8], although most of the global decline may be attributed to these species, with greater importance in terms of ecosystem structure, function and services provided [8-10]. In some cases, the measures that could help endangered and common species are identical, but that is not always the case, so investigations to determine the intrinsic or extrinsic traits most strongly associated with significant declines of common species are urgently needed [8].

Species trend indicators are considered a sensitive meas-

*Corresponding author: Consejería de Medio Ambiente, Junta de Castilla y León, C/ Rigoberto Cortejoso, 1447071 Valladolid, Spain

Accepted: November 09, 2020

Published online: November 11, 2020

Citation: Balmori A, Balmori-de la Puente A (2020) Testing if Habitat and Movements Determine the Threats and Trends for Common Birds in Europe. Adv Environ Stud 4(2):331-344 
ure of biodiversity change [4]. Such indicators can provide information for decision makers and improve the management of natural resources [6]. For this reason, the results of projects monitoring common species should be made available to policy-makers, their advisors and the general public [7].

Birds are often regarded as good and useful indicators of biodiversity for other elements of biodiversity due to the fact that they are well known and studied and are very sensitive to anthropogenic changes $[5,9]$. Trends in bird populations correlate in some situations with those of other taxa, making them a valuable biodiversity indicators; however, it is necessary to take into account that birds are usually very mobile and integrate environmental changes over huge areas, so further analysis should be done to explore the temporal and spatial correlations across taxa trends in different areas $[3,5,6]$.

Wild bird indicators have been adopted by the European Union as indicators of biodiversity and sustainable development. These bird indicators are studied within a consortium of organisations from many countries through the European Bird Census Council (EBCC) to measure breeding population changes [5]. The specific aim of these indicators was approaching to assess the mean change in breeding bird populations of European farmland and woodland (including woods, parks and gardens), because these habitats represent the predominant land use types in Europe [4-6].

European populations of several birds, especially farmland birds, are declining alarmingly, mainly due to agricultural modernisation and intensification [5,6,8-11]. Birds living in forests have declined on average by $14 \%$, while common birds living in farmland have fallen steeply, by $43 \%$ in 25 years [6]. Studies in Europe have shown that many vertebrate, insect and plant species of farmland have declined in parallel, driven by agricultural intensification and specialisation $[4,5]$. In fact, farmland species have experienced much greater population declines in three decades in the 15 member countries of the EU than in the 10 recently acceded countries (May 2004) or in the remaining 18 European countries that do not belong to the European Union [4].

The aim of this work was to analyse and compare the main threats and population trends for common birds in Europe given their habitats and movements (migratory or sedentary character), to study the existence of possible relationships between the different variables. This is therefore a theoretical study that creates a database that gathers this information in order to investigate the specific factors associated with the threats and population trends of common birds, and in general the relationships between these four variables that condition in an important way the state of their populations, and can be of help for the adoption of specific conservation measures for common species in decline.

\section{Material and Methods}

The List of 167 European common bird species that were included in the analysis was extracted from "All common bird indicators in Europe": http://www.ebcc.info/. European species selection/classification have been based on expert judgment. Regional coordinators were responsible for the production of regional species lists in cooperation with all rel- evant experts within their regions $h$ ttps://pecbms.info/methods/pecbms-methods/3-multispecies-indicators/species-selection-and-classification/

\section{Habitats}

For each common species, information on their habitat was extracted from Nicolai, et al. [12]. For common species who live in several different types of habitats, the one that was considered most significant and frequent for each species was chosen. According to their habitat, the birds were classified into six categories; Farmland (including Open countryside, Meadows and Orchards), Forest (Including Schrubs), Wetlands (including Rivers), Mountains (Including Cliffs), Urban (Including Gardens and Parkland) and Coastal fields (Table $1 \mathrm{a}$, Table $1 \mathrm{~b}$ and Table $1 \mathrm{c})$.

\section{Movements}

The common birds were classified into three categories following Peterson, et al. [13]: Sedentary, Partial migration and Aestival. Although in many cases mixed situations within the same species are found among these three types of movement patterns, the most common or dominant character for each species in Europe was chosen (Table 1a, Table 1b and Table 1c), following these criteria:

a) Aestival: Migrant species with trans-Saharan wintering areas.

b) Sedentary: The majority of the population remains in the breeding area during the winter.

c) Partial migration: Includes species with winter movements of a part or the majority of the European population but staying in southern Europe or North Africa (without crossing the Sahara desert: Pre-Saharan).

\section{Threats}

The threats for each common species were obtained from the Atlas of European Breeding birds [14] and the IUCN website: http://www.iucnredlist.org/

Each species may have several threats that can affect its populations to different degrees, some severely, others slightly, and harmfulness can differ depending on location. Thus, for statistical analysis the threat considered most harmful for each species was chosen. The most harmful threat for each species, was determined considering the most important in its entire range, being generally the one mentioned first in the Atlas of European Breeding birds and the IUCN. Initially, the different threats were classified into 20 categories but finally, in order to simplify the statistical analysis, the threats were grouped in six categories as follows: Climatic factors and Contamination, Competition (including Predation and Lack of food), Human disturbance (including Fishing, Lead poisoning, Poisoning, Power lines, Run over, Spoliation of nests, Trapping and hunting, Wind farms accident), Habitat loss (including Forest fires), Diseases (including Genetic problems), Invasive species and "Without any known threat" (the last not considered in the statistical analysis) (Table 1a, Table $1 b$ and Table 1c). The criteria for inclusion in each threat category are presented in Annex 1. 
Citation: Balmori A, Balmori-de la Puente A (2020) Testing if Habitat and Movements Determine the Threats and Trends for Common Birds in Europe. Adv Environ Stud 4(2):331-344

Table 1a: Habitats, movements, threats, trends and base year trends for the 167 European common birds considered in this work.

\begin{tabular}{|c|c|c|c|c|c|}
\hline Species & Habitat & Movements & Threats & Trends & $\begin{array}{l}\text { Base year for } \\
\text { trends }\end{array}$ \\
\hline Accipiter nisus & Forest & Partial migration & Human disturbance & Stable & 1980 \\
\hline Acrocephalus arundinaceus & Wetlands & Aestival & Habitat loss & Moderate increase & 1982 \\
\hline Acrocephalus palustris & Wetlands & Aestival & $\begin{array}{l}\text { Climatic factors and } \\
\text { Contamination }\end{array}$ & Stable & 1980 \\
\hline $\begin{array}{l}\text { Acrocephalus } \\
\text { schoenobaenus }\end{array}$ & Wetlands & Aestival & Habitat loss & Stable & 1980 \\
\hline Acrocephalus scirpaceus & Wetlands & Aestival & Habitat loss & Stable & 1980 \\
\hline Actitis hypoleucos & Wetlands & Aestival & Habitat loss & Moderate decline & 1980 \\
\hline Aegithalos caudatus & Forest & Partial migration & Habitat loss & Stable & 1980 \\
\hline Alauda arvensis & Farmland & Partial migration & Competition & Moderate decline & 1980 \\
\hline Alcedo atthis & Wetlands & Partial migration & Habitat loss & Stable & 1991 \\
\hline Alectoris rufa & Farmland & Sedentary & Habitat loss & Moderate decline & 1998 \\
\hline Anas platyrhynchos & Wetlands & Partial migration & Habitat loss & Moderate increase & 1980 \\
\hline Anthus campestris & Farmland & Aestival & Habitat loss & Uncertain & 1991 \\
\hline Anthus pratensis & Farmland & Partial migration & $\begin{array}{l}\text { Climatic factors and } \\
\text { Contamination }\end{array}$ & Moderate decline & 1980 \\
\hline Anthus trivialis & Forest & Aestival & Habitat loss & Moderate decline & 1980 \\
\hline Apus apus & Urban & Aestival & $\begin{array}{l}\text { Climatic factors and } \\
\text { Contamination }\end{array}$ & Stable & 1980 \\
\hline Ardea cinerea & Wetlands & Partial migration & Human disturbance & Moderate increase & 1980 \\
\hline Bombycilla garrulus & Forest & Partial migration & Habitat loss & Strong increase & 1988 \\
\hline Bonasa bonasia & Forest & Sedentary & Human disturbance & Stable & 1980 \\
\hline Bubulcus ibis & Farmland & Sedentary & Habitat loss & Stable & 1998 \\
\hline Burhinus oedicnemus & Farmland & Partial migration & Habitat loss & Stable & 1998 \\
\hline Buteo buteo & Forest & Partial migration & Human disturbance & Moderate increase & 1980 \\
\hline Calandrella brachydactyla & Farmland & Aestival & Habitat loss & Stable & 1998 \\
\hline Calcarius lapponicus & Mountains & Partial migration & $\begin{array}{l}\text { Climatic factors and } \\
\text { Contamination }\end{array}$ & Uncertain & 2000 \\
\hline Carduelis cannabina & Farmland & Partial migration & Competition & Moderate decline & 1980 \\
\hline Carduelis carduelis & Farmland & Partial migration & Without any known threat & Moderate increase & 1980 \\
\hline Carduelis chloris & Forest & Partial migration & Without any known threat & Stable & 1980 \\
\hline Carduelis flammea & Forest & Partial migration & Competition & Moderate decline & 1980 \\
\hline Carduelis spinus & Forest & Partial migration & Competition & Moderate decline & 1980 \\
\hline Carpodacus erythrinus & Forest & Aestival & Without any known threat & Moderate decline & 1980 \\
\hline Certhia brachydactyla & Forest & Sedentary & Habitat loss & Moderate increase & 1982 \\
\hline Certhia familiaris & Forest & Sedentary & $\begin{array}{l}\text { Climatic factors and } \\
\text { Contamination }\end{array}$ & Stable & 1980 \\
\hline Cettia cetti & Wetlands & Sedentary & $\begin{array}{l}\text { Climatic factors and } \\
\text { Contamination }\end{array}$ & Moderate increase & 1989 \\
\hline Ciconia ciconia & Wetlands & Aestival & Habitat loss & Moderate increase & 1980 \\
\hline Circus aeruginosus & Wetlands & Partial migration & $\begin{array}{l}\text { Climatic factors and } \\
\text { Contamination }\end{array}$ & Moderate increase & 1980 \\
\hline Cisticola juncidis & Wetlands & Sedentary & $\begin{array}{l}\text { Climatic factors and } \\
\text { Contamination }\end{array}$ & Stable & 1998 \\
\hline Clamator glandarius & Farmland & Aestival & Without any known threat & Moderate increase & 1998 \\
\hline $\begin{array}{l}\text { Coccothraustes } \\
\text { coccothraustes }\end{array}$ & Forest & Partial migration & Habitat loss & Moderate increase & 1980 \\
\hline
\end{tabular}


Citation: Balmori A, Balmori-de la Puente A (2020) Testing if Habitat and Movements Determine the Threats and Trends for Common Birds in Europe. Adv Environ Stud 4(2):331-344

\begin{tabular}{|c|c|c|c|c|c|}
\hline Columba oenas & Forest & Partial migration & Competition & Moderate increase & 1980 \\
\hline Columba palumbus & Forest & Partial migration & $\begin{array}{l}\text { Climatic factors and } \\
\text { Contamination }\end{array}$ & Moderate increase & 1980 \\
\hline Corvus corax & Forest & Sedentary & Habitat loss & Moderate increase & 1980 \\
\hline Corvus corone & Farmland & Sedentary & Without any known threat & Moderate increase & 1980 \\
\hline Corvus frugilegus & Farmland & Partial migration & Habitat loss & Moderate increase & 1980 \\
\hline Corvus monedula & Urban & Partial migration & Without any known threat & Stable & 1980 \\
\hline Cuculus canorus & Farmland & Aestival & Habitat loss & Moderate decline & 1980 \\
\hline Cyanopica cyanus & Forest & Sedentary & Competition & Moderate increase & 1998 \\
\hline Cygnus olor & Wetlands & Partial migration & Human disturbance & Moderate increase & 1980 \\
\hline Delichon urbica & Urban & Aestival & $\begin{array}{l}\text { Climatic factors and } \\
\text { Contamination }\end{array}$ & Moderate decline & 1980 \\
\hline Dendrocopos major & Forest & Sedentary & $\begin{array}{l}\text { Climatic factors and } \\
\text { Contamination }\end{array}$ & Moderate increase & 1980 \\
\hline Dendrocopos medius & Forest & Sedentary & Habitat loss & Stable & 1989 \\
\hline Dendrocopos minor & Forest & Sedentary & Habitat loss & Uncertain & 1980 \\
\hline Dendrocopos syriacus & Forest & Sedentary & Without any known threat & Moderate decline & 1999 \\
\hline Dryocopus martius & Forest & Sedentary & Habitat loss & Moderate increase & 1980 \\
\hline Egretta garzetta & Wetlands & Partial migration & Habitat loss & Stable & 2000 \\
\hline Emberiza cia & Farmland & Sedentary & Habitat loss & Moderate increase & 1998 \\
\hline Emberiza cirlus & Farmland & Sedentary & Habitat loss & Moderate increase & 1989 \\
\hline
\end{tabular}

Table 1b: Habitats, movements, threats, trends and base year trends for the 167 European common birds considered in this work.

\begin{tabular}{|c|c|c|c|c|c|}
\hline Species & Habitat & Movements & Threats & Trends & $\begin{array}{l}\text { Base year for } \\
\text { trends }\end{array}$ \\
\hline Emberiza citrinella & Farmland & Partial migration & Habitat loss & Moderate decline & 1980 \\
\hline Emberiza hortulana & Farmland & Aestival & Habitat loss & Steep decline & 1980 \\
\hline $\begin{array}{l}\text { Emberiza } \\
\text { melanocephala }\end{array}$ & Farmland & Aestival & Habitat loss & Steep decline & 2000 \\
\hline Emberiza rustica & Forest & Aestival & Without any known threat & Moderate decline & 1980 \\
\hline Emberiza schoeniclus & Wetlands & Partial migration & Habitat loss & Moderate decline & 1980 \\
\hline Erithacus rubecula & Forest & Partial migration & Climatic factors and Contamination & Moderate increase & 1980 \\
\hline Falco tinnunculus & Farmland & Partial migration & Climatic factors and Contamination & Moderate decline & 1980 \\
\hline Ficedula albicollis & Forest & Aestival & Habitat loss & Moderate increase & 1982 \\
\hline Ficedula hypoleuca & Forest & Aestival & Climatic factors and Contamination & Moderate decline & 1980 \\
\hline Fringilla coelebs & Forest & Partial migration & Climatic factors and Contamination & Moderate increase & 1980 \\
\hline Fringilla montifringilla & Forest & Partial migration & Climatic factors and Contamination & Moderate decline & 1980 \\
\hline Fulica atra & Wetlands & Partial migration & Human disturbance & Moderate increase & 1980 \\
\hline Galerida cristata & Farmland & Sedentary & Habitat loss & Steep decline & 1982 \\
\hline Galerida theklae & Farmland & Sedentary & Habitat loss & Moderate increase & 1998 \\
\hline Gallinago gallinago & Wetlands & Partial migration & Habitat loss & Moderate decline & 1980 \\
\hline Gallinula chloropus & Wetlands & Partial migration & Climatic factors and Contamination & Stable & 1980 \\
\hline Garrulus glandarius & Forest & Partial migration & Without any known threat & Moderate increase & 1980 \\
\hline Grus grus & Wetlands & Partial migration & Habitat loss & Moderate increase & 1984 \\
\hline $\begin{array}{l}\text { Haematopus } \\
\text { ostralegus }\end{array}$ & Coastal fields & Partial migration & Habitat loss & Moderate decline & 1980 \\
\hline Hippolais icterina & Forest & Aestival & Habitat loss & Moderate decline & 1980 \\
\hline Hippolais polyglotta & Urban & Aestival & Without any known threat & Stable & 1989 \\
\hline
\end{tabular}


Citation: Balmori A, Balmori-de la Puente A (2020) Testing if Habitat and Movements Determine the Threats and Trends for Common Birds in Europe. Adv Environ Stud 4(2):331-344

\begin{tabular}{|c|c|c|c|c|c|}
\hline Hirundo daurica & Urban & Aestival & Without any known threat & Stable & 1998 \\
\hline Hirundo rupestris & Mountains & Partial migration & Without any known threat & Stable & 1998 \\
\hline Hirundo rustica & Farmland & Aestival & Competition & Stable & 1980 \\
\hline Jynx torquilla & Forest & Aestival & Climatic factors and Contamination & Moderate decline & 1980 \\
\hline Lanius collurio & Forest & Aestival & Climatic factors and Contamination & Stable & 1980 \\
\hline Lanius minor & Forest & Aestival & Climatic factors and Contamination & Moderate decline & 1999 \\
\hline Lanius senator & Forest & Aestival & Climatic factors and Contamination & Moderate decline & 1998 \\
\hline Larus ridibundus & Coastal fields & Partial migration & Climatic factors and Contamination & Moderate decline & 1990 \\
\hline Limosa limosa & Coastal fields & Partial migration & Habitat loss & Moderate decline & 1984 \\
\hline Locustella fluviatilis & Wetlands & Aestival & Climatic factors and Contamination & Moderate decline & 1982 \\
\hline Locustella naevia & Farmland & Aestival & Climatic factors and Contamination & Stable & 1980 \\
\hline Lullula arborea & Forest & Aestival & Habitat loss & Moderate increase & 1980 \\
\hline Luscinia luscinia & Forest & Aestival & Habitat loss & Stable & 1980 \\
\hline $\begin{array}{l}\text { Luscinia } \\
\text { megarhynchos }\end{array}$ & Forest & Aestival & Habitat loss & Moderate decline & 1980 \\
\hline $\begin{array}{l}\text { Luscinia svecica } \\
\text { svecica }\end{array}$ & Farmland & Aestival & Habitat loss & Moderate decline & 1996 \\
\hline $\begin{array}{l}\text { Melanocorypha } \\
\text { calandra }\end{array}$ & Farmland & Sedentary & Habitat loss & Moderate decline & 1998 \\
\hline Merops apiaster & Farmland & Aestival & Habitat loss & Uncertain & 1989 \\
\hline Miliaria calandra & Farmland & Partial migration & Habitat loss & Moderate decline & 1980 \\
\hline Motacilla alba & Wetlands & Partial migration & Climatic factors and Contamination & Stable & 1980 \\
\hline Motacilla cinerea & Wetlands & Partial migration & Climatic factors and Contamination & Stable & 1980 \\
\hline Motacilla flava & Farmland & Aestival & Habitat loss & Moderate decline & 1980 \\
\hline Muscicapa striata & Forest & Aestival & Climatic factors and Contamination & Moderate decline & 1980 \\
\hline $\begin{array}{l}\text { Nucifraga } \\
\text { caryocatactes }\end{array}$ & Forest & Sedentary & Habitat loss & Stable & 1980 \\
\hline Numenius arquata & Coastal fields & Partial migration & Habitat loss & Moderate decline & 1980 \\
\hline Numenius phaeopus & Coastal fields & Partial migration & Competition & Stable & 1984 \\
\hline Oenanthe hispanica & Farmland & Aestival & Climatic factors and Contamination & Moderate decline & 1998 \\
\hline Oenanthe oenanthe & Farmland & Aestival & Habitat loss & Moderate decline & 1980 \\
\hline Oriolus oriolus & Forest & Aestival & Climatic factors and Contamination & Stable & 1982 \\
\hline Parus ater & Forest & Partial migration & Competition & Moderate decline & 1980 \\
\hline Parus caeruleus & Forest & Partial migration & Without any known threat & Moderate increase & 1980 \\
\hline Parus cristatus & Forest & Sedentary & Climatic factors and Contamination & Moderate decline & 1980 \\
\hline Parus major & Forest & Partial migration & Competition & Moderate increase & 1980 \\
\hline Parus montanus & Forest & Sedentary & Without any known threat & Moderate decline & 1980 \\
\hline Parus palustris & Forest & Sedentary & Habitat loss & Moderate decline & 1980 \\
\hline
\end{tabular}

\section{Species trends}

The information on the trends was extracted from "All common bird indicators in Europe": http://www.ebcc.info/

The trends of the 167 European common birds were classified into six categories: Steep increasing, moderate increasing, stable, moderate declining, steep declining and uncertain (Table 1a, Table $1 \mathrm{~b}$ and Table 1c). But in order to simplify the statistical analysis, they were reduced to three categories (increase, decline and stable) as follows: "Uncertain" was not considered; steep increasing and moderate increasing both were considered as "increase", moderate and steep decline both were considered as "decline" and stable species remain as "stable". For 104 birds, information about species trends has been available since 1980. For 27 species, since the 1980s, for 31 species since the 1990s and for five species since 2000 (Table 1a, Table 1b and Table 1c).

\section{Database and statistics}

All websites were consulted in January 2016. A database with all trends, threats, habitats and movements for the 167 common bird species considered in this study was prepared 
Citation: Balmori A, Balmori-de la Puente A (2020) Testing if Habitat and Movements Determine the Threats and Trends for Common Birds in Europe. Adv Environ Stud 4(2):331-344

Table 1c: Habitats, movements, threats, trends and base year trends for the 167 European common birds considered in this work.

\begin{tabular}{|c|c|c|c|c|c|}
\hline Species & Habitat & Movements & Threats & Threats Trends & $\begin{array}{l}\text { Base year for } \\
\text { trends }\end{array}$ \\
\hline Passer domesticus & Urban & Sedentary & Habitat loss & Moderate decline & 1980 \\
\hline Passer montanus & Farmland & Partial migration & Competition & Moderate decline & 1980 \\
\hline Perdix perdix & Farmland & Sedentary & Habitat loss & Steep decline & 1980 \\
\hline Petronia petronia & Farmland & Sedentary & Without any known threat & Stable & 1998 \\
\hline Phasianus colchicus & Farmland & Sedentary & Competition & Moderate increase & 1980 \\
\hline Phoenicurus ochruros & Mountains & Partial migration & Without any known threat & Moderate increase & 1982 \\
\hline $\begin{array}{l}\text { Phoenicurus } \\
\text { phoenicurus }\end{array}$ & Forest & Aestival & Climatic factors and Contamination & Moderate increase & 1980 \\
\hline Phylloscopus bonelli & Forest & Aestival & Without any known threat & Stable & 1989 \\
\hline Phylloscopus collybita & Forest & Partial migration & Habitat loss & Moderate increase & 1980 \\
\hline Phylloscopus sibilatrix & Forest & Aestival & Climatic factors and Contamination & Moderate decline & 1980 \\
\hline Phylloscopus trochilus & Forest & Aestival & Without any known threat & Moderate decline & 1980 \\
\hline Pica pica & Farmland & Sedentary & Competition & Moderate decline & 1980 \\
\hline Picus canus & Forest & Sedentary & Climatic factors and Contamination & Moderate increase & 1982 \\
\hline Picus viridis & Forest & Sedentary & Habitat loss & Moderate increase & 1980 \\
\hline Pluvialis apricaria & $\begin{array}{l}\text { Coastal } \\
\text { fields }\end{array}$ & Partial migration & Habitat loss & Stable & 1981 \\
\hline Podiceps cristatus & Wetlands & Partial migration & Climatic factors and Contamination & Moderate decline & 1990 \\
\hline Prunella modularis & Forest & Partial migration & Habitat loss & Moderate decline & 1980 \\
\hline $\begin{array}{l}\text { Pyrrhocorax } \\
\text { pyrrhocorax }\end{array}$ & Mountains & Sedentary & Habitat loss & Stable & 1998 \\
\hline Pyrrhula pyrrhula & Forest & Sedentary & Competition & Moderate decline & 1980 \\
\hline Regulus ignicapilla & Forest & Partial migration & Without any known threat & Stable & 1982 \\
\hline Regulus regulus & Forest & Partial migration & Climatic factors and Contamination & Moderate decline & 1980 \\
\hline Saxicola rubetra & Farmland & Aestival & Without any known threat & Moderate decline & 1980 \\
\hline Saxicola torquata & Farmland & Partial migration & Habitat loss & Stable & 1989 \\
\hline Serinus citrinella & Forest & Sedentary & Without any known threat & Moderate decline & 2002 \\
\hline Serinus serinus & Forest & Partial migration & Without any known threat & Moderate decline & 1982 \\
\hline Sitta europaea & Forest & Sedentary & Habitat loss & Moderate increase & 1980 \\
\hline Streptopelia decaocto & Urban & Sedentary & Without any known threat & Moderate increase & 1980 \\
\hline Streptopelia turtur & Farmland & Aestival & Habitat loss & Moderate decline & 1980 \\
\hline Sturnus unicolor & Farmland & Sedentary & Without any known threat & Moderate increase & 1998 \\
\hline Sturnus vulgaris & Farmland & Partial migration & Habitat loss & Moderate decline & 1980 \\
\hline Sylvia atricapilla & Forest & Partial migration & Without any known threat & Moderate increase & 1980 \\
\hline Sylvia borin & Forest & Aestival & Climatic factors and Contamination & Moderate decline & 1980 \\
\hline Sylvia cantillans & Forest & Aestival & Without any known threat & Moderate increase & 1989 \\
\hline Sylvia communis & Forest & Aestival & Climatic factors and Contamination & Moderate increase & 1980 \\
\hline Sylvia curruca & Urban & Aestival & Habitat loss & Stable & 1980 \\
\hline Sylvia hortensis & Urban & Aestival & Habitat loss & Moderate increase & 1989 \\
\hline Sylvia melanocephala & Forest & Sedentary & Without any known threat & Stable & 1989 \\
\hline Sylvia nisoria & Forest & Aestival & Climatic factors and Contamination & Uncertain & 1982 \\
\hline Sylvia undata & Forest & Sedentary & Habitat loss & Moderate decline & 1998 \\
\hline Tachybaptus ruficollis & Wetlands & Partial migration & Climatic factors and Contamination & Stable & 1990 \\
\hline Tadorna tadorna & Wetlands & Partial migration & Habitat loss & Moderate increase & 1991 \\
\hline
\end{tabular}


Citation: Balmori A, Balmori-de la Puente A (2020) Testing if Habitat and Movements Determine the Threats and Trends for Common Birds in Europe. Adv Environ Stud 4(2):331-344

\begin{tabular}{|c|c|c|c|c|c|}
\hline Tetrao tetrix & Farmland & Sedentary & Habitat loss & Stable & 1996 \\
\hline Tetrax tetrax & Farmland & Partial migration & Habitat loss & Moderate decline & 1998 \\
\hline Tringa erythropus & Wetlands & Partial migration & Habitat loss & Moderate decline & 2006 \\
\hline Tringa glareola & Wetlands & Partial migration & Habitat loss & Stable & 1980 \\
\hline Tringa nebularia & Farmland & Partial migration & Habitat loss & Stable & 1998 \\
\hline Tringa ochropus & Wetlands & Partial migration & Diseases & Moderate increase & 1980 \\
\hline Tringa totanus & Wetlands & Partial migration & Habitat loss & Moderate decline & 1980 \\
\hline $\begin{array}{l}\text { Troglodytes } \\
\text { troglodytes }\end{array}$ & Wetlands & Partial migration & Climatic factors and Contamination & Moderate increase & 1980 \\
\hline Turdus iliacus & Forest & Partial migration & Climatic factors and Contamination & Moderate decline & 1980 \\
\hline Turdus merula & Forest & Partial migration & Climatic factors and Contamination & Moderate increase & 1980 \\
\hline Turdus philomelos & Forest & Partial migration & Climatic factors and Contamination & Moderate increase & 1980 \\
\hline Turdus pilaris & Forest & Partial migration & Without any known threat & Stable & 1980 \\
\hline Turdus torquatus & Forest & Partial migration & Human disturbance & Stable & 1998 \\
\hline Turdus viscivorus & Forest & Partial migration & Habitat loss & Moderate decline & 1980 \\
\hline Upupa epops & Farmland & Aestival & Climatic factors and Contamination & Uncertain & 1982 \\
\hline Vanellus vanellus & Wetlands & Partial migration & Habitat loss & Moderate decline & 1980 \\
\hline
\end{tabular}

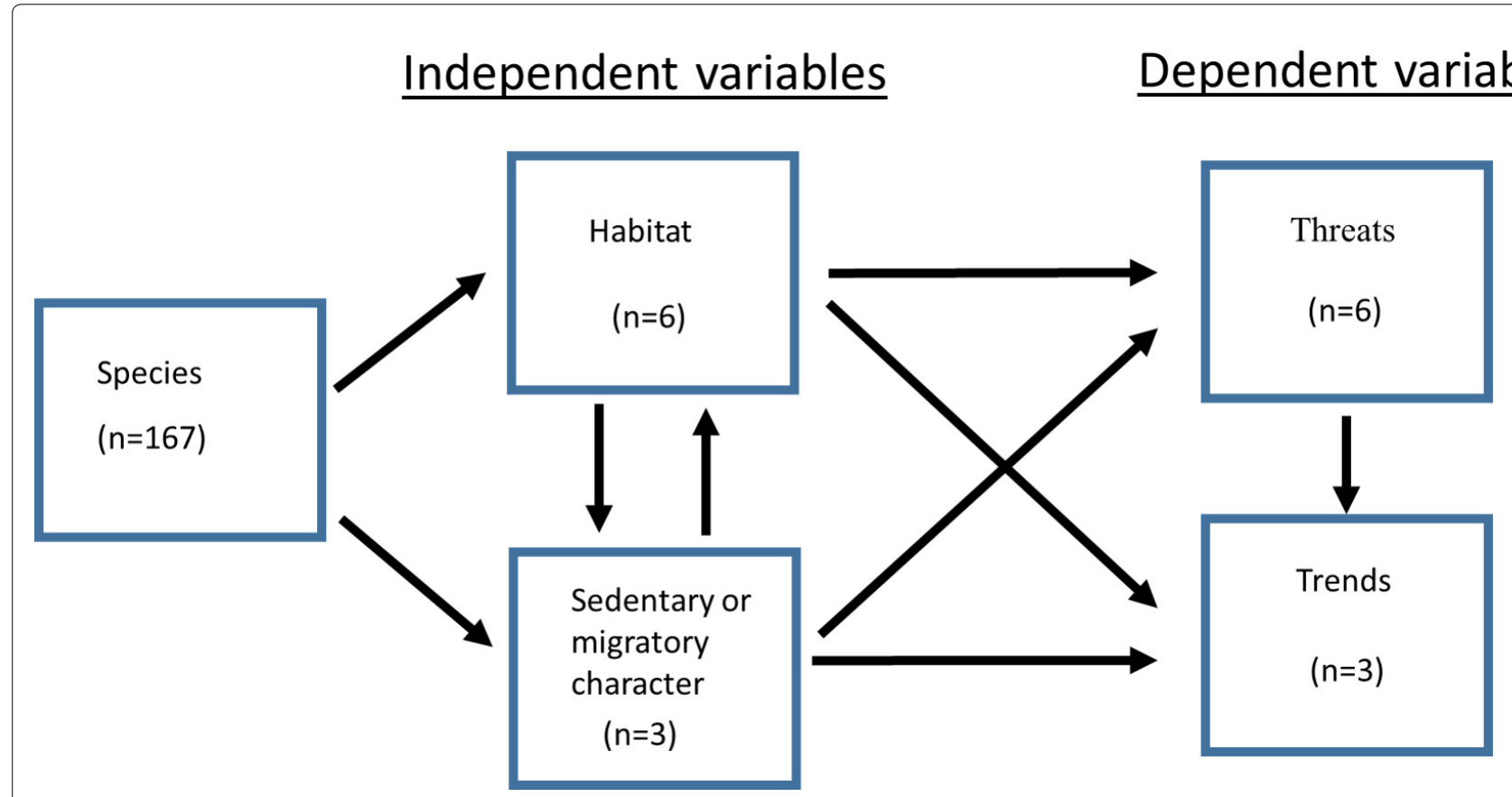

Figure 1: Structure of variables for the statistical analysis used in this work.

in Access 2013. The organization of information for statistical analysis is shown in Figure 1, although all the possible interactions between variables were checked as in log-linear and contingency tables analysis there is no need to specify which are the predictors. In a first attempt to perform statistical analysis, the four categorical variables (habitats, movements, threats and trends) were checked together in order to find associations or some relationships between them, using a log-linear analysis with the GLM function in R. Afterward, groups of three variables were checked for independence following the procedure described above. Finally, contingency tables considering two categorical variables in each case were performed. In these two-way analysis the Fisher test, which is recommended for datasets with small expected numbers for each combination cell, was used. All statistical analyses were performed using R program [15].

\section{Results}

The list of 167 common species considered in this work and their habitats, movements, threats, trends and base year for trends are presented in Table 1a, Table $1 \mathrm{~b}$ and Table $1 \mathrm{c}$.

The summary of their habitat is: Farmland $(n=44)$ Forest $(n=73)$, Wetlands $(n=31)$, Mountains $(n=4)$, Urban $(n=9)$ and Coastal fields $(n=6)$. 
The summary of movements is Partial migration $(n=75)$, Aestival $(n=52)$ and Sedentary $(n=40)$.

Regarding the threats, we had information for 139 species and no such information for 28 species (not included in the statistical analyses). The most frequent threat was "Habitat loss", ( $n=73$ ), followed by "Climatic factors and contamination" ( $n=44)$, "Competition" ( $=14)$ "Human disturbance" ( $n=7$ ) and "Diseases" ( $n=1$ ). Invasive species, although is a serious threat to the whole biodiversity, but it was not the most important threat to any particular species.

The summary of trends is as follow: 51 species with "increase" trend (27 Forest, 9 Farmland, 1 Mountains, 2 Urban, 12 Wetlands); 44 species with "stable" trend (15 Forest, 9 Farmland, 2 Coastal fields, 2 Mountains, 5 Urban and 11 Wetlands); 65 species with "decline" trend (29 Forest, 22 Farmland, 4 Coastal fields, 2 Urban and 8 Wetlands). Finally, seven species with "uncertain" trend (2 Forest, 4 Farmland, 1 Mountains) but not considered in the analysis.

\section{Log linear four-dimension analysis}

In the performed analysis including the four variables there was not found any statistically significant results.

\section{Log linear three-dimension analysis}

Taking the variables studied by groups of three, the combination: "Habitats $\times$ Movements $\times$ Threats" has its residual deviance with a marginal statistical significance ( $p=0.07$; Table 2 ). We can observe that expected values for the model assuming independency of variables do not fit with observed ones, especially in the cases of "Forest - Aestival - Climatic factors and Contamination" 5.6 and 12 respectively and "Farmland - Sedentary - Habitat loss" 4.5 and 9 respectively which are underestimated by the model and "Forest - Partial migration - Habitat loss" 13.8 and 6 respectively, which is overestimated by the model (highlighted in Table 2). This indicates that Forest - Aestival species and Farmland- Sedentary ones are affected by Climatic factors and contamination and Habitat loss respectively more than expected, whereas Forest species with Partial migration are less affected by Habitat loss than should be.

The combination "Habitats $\times$ Movements $\times$ Trends" have a high statistical significance ( $p=0.009$; Table 3$)$ which indicates a poor fit of the model assuming that the three variables are independent between them. This provokes again that our expected value with the model does not fit with the observed data, especially in cases of "Forest - Aestival - Decline" 8.5 and 13 respectively and "Wetlands - Partial migration - Increase" 4.6 and 9 respectively which are underestimated by the model and "Forest - Partial migration - Decline" 13.3 and 9 respectively which is overestimated (highlighted in the Table 3). This indicates that Forest - Aestival species are declining more than expected, that Wetland - Partial migration ones are increasing more than expected, and that Forest - Partial migration species are declining less than predicted by the random model.

In the rest of the possible three-dimension combinations

Table 2: Log linear analyses of habitats, movements and threats showing observed (Freq) and expected (Fitted) values obtained with the model assuming the three variables are independent of each other $(p=0.07)$. Note that highest differences between observed and expected values are in bold.

\begin{tabular}{|c|c|c|c|c|c|c|c|c|c|c|c|}
\hline & & \multicolumn{2}{|c|}{$\begin{array}{l}\text { Climatic factors and } \\
\text { Contamination }\end{array}$} & \multicolumn{2}{|c|}{ Competition } & \multicolumn{2}{|c|}{ Diseases } & \multicolumn{2}{|c|}{ Habitat loss } & \multicolumn{2}{|c|}{$\begin{array}{l}\text { Human } \\
\text { disturbance }\end{array}$} \\
\hline & & Freq & Fitted & Freq & Fitted & Freq & Fitted & Freq & Fitted & Freq & Fitted \\
\hline Coastalfields & Aestival & 0 & 0.6 & 0 & 0.2 & 0 & 0 & 0 & 1 & 0 & 0.1 \\
\hline Farmland & Aestival & 3 & 3.6 & 1 & 1.2 & 0 & 0.1 & 9 & 6 & 0 & 0.6 \\
\hline Forest & Aestival & 12 & 5.6 & 0 & 1.8 & 0 & 0.1 & 6 & 9.3 & 0 & 0.9 \\
\hline Mountains & Aestival & 0 & 0.2 & 0 & 0.1 & 0 & 0 & 0 & 0.3 & 0 & 0 \\
\hline Urban & Aestival & 2 & 0.6 & 0 & 0.2 & 0 & 0 & 3 & 1 & 0 & 0.1 \\
\hline Wetlands & Aestival & 2 & 3 & 0 & 1 & 0 & 0.1 & 5 & 5 & 0 & 0.5 \\
\hline Coastalfields & Partial migration & 1 & 0.9 & 1 & 0.3 & 0 & 0 & 4 & 1.5 & 0 & 0.1 \\
\hline Farmland & Partial migration & 2 & 5.4 & 3 & 1.7 & 0 & 0.1 & 8 & 8.9 & 0 & 0.9 \\
\hline Forest & Partial migration & 8 & 8.3 & 5 & 2.6 & 0 & 0.2 & 6 & 13.8 & 3 & 1.3 \\
\hline Mountains & Partial migration & 1 & 0.3 & 0 & 0.1 & 0 & 0 & 0 & 0.5 & 0 & 0 \\
\hline Urban & Partial migration & 0 & 0.9 & 0 & 0.3 & 0 & 0 & 0 & 1.5 & 0 & 0.1 \\
\hline Wetlands & Partial migration & 7 & 4.5 & 0 & 1.4 & 1 & 0.1 & 11 & 7.5 & 3 & 0.7 \\
\hline Coastalfields & Sedentary & 0 & 0.4 & 0 & 0.1 & 0 & 0 & 0 & 0.7 & 0 & 0.1 \\
\hline Farmland & Sedentary & 0 & 2.7 & 2 & 0.9 & 0 & 0.1 & 9 & 4.5 & 0 & 0.4 \\
\hline Forest & Sedentary & 4 & 4.2 & 2 & 1.3 & 0 & 0.1 & 10 & 6.9 & 1 & 0.7 \\
\hline Mountains & Sedentary & 0 & 0.1 & 0 & 0 & 0 & 0 & 1 & 0.2 & 0 & 0 \\
\hline Urban & Sedentary & 0 & 0.4 & 0 & 0.1 & 0 & 0 & 1 & 0.7 & 0 & 0.1 \\
\hline Wetlands & Sedentary & 2 & 2.3 & 0 & 0.7 & 0 & 0.1 & 0 & 3.7 & 0 & 0.4 \\
\hline
\end{tabular}


Citation: Balmori A, Balmori-de la Puente A (2020) Testing if Habitat and Movements Determine the Threats and Trends for Common Birds in Europe. Adv Environ Stud 4(2):331-344

Table 3: Log linear analyses of habitats, movements and trends showing observed (Freq) and expected (Fitted) values obtained with the model assuming the three variables are independent of each other $(p=0.009)$. Note that highest differences between observed and expected values are in bold.

\begin{tabular}{|c|c|c|c|c|c|c|c|}
\hline & & \multicolumn{2}{|c|}{ Decline } & \multicolumn{2}{|c|}{ Increase } & \multicolumn{2}{|c|}{ Stable } \\
\hline & & Freq & Fitted & Freq & Fitted & Freq & Fitted \\
\hline Coastalfields & Aestival & 0 & 0.7 & 0 & 0.6 & 0 & 0.5 \\
\hline Farmland & Aestival & 7 & 4.7 & 1 & 3.7 & 3 & 3.2 \\
\hline Forest & Aestival & 13 & 8.5 & 5 & 6.6 & 4 & 5.7 \\
\hline Mountains & Aestival & 0 & 0.4 & 0 & 0.3 & 0 & 0.2 \\
\hline Urban & Aestival & 2 & 1.2 & 1 & 0.9 & 4 & 0.8 \\
\hline Wetlands & Aestival & 2 & 3.7 & 2 & 2.9 & 3 & 2.5 \\
\hline Coastalfields & Partial migration & 4 & 1.1 & 0 & 0.9 & 2 & 0.8 \\
\hline Farmland & Partial migration & 9 & 7.3 & 2 & 5.7 & 3 & 5 \\
\hline Forest & Partial migration & 9 & 13.3 & 14 & 10.5 & 6 & 9 \\
\hline Mountains & Partial migration & 0 & 0.6 & 1 & 0.4 & 1 & 0.4 \\
\hline Urban & Partial migration & 0 & 1.9 & 0 & 1.5 & 1 & 1.3 \\
\hline Wetlands & Partial migration & 6 & 5.8 & 9 & 4.6 & 7 & 3.9 \\
\hline Coastalfields & Sedentary & 0 & 0.6 & 0 & 0.5 & 0 & 0.4 \\
\hline Farmland & Sedentary & 5 & 3.9 & 6 & 3 & 3 & 2.6 \\
\hline Forest & Sedentary & 7 & 7 & 8 & 5.5 & 5 & 4.8 \\
\hline Mountains & Sedentary & 0 & 0.3 & 0 & 0.2 & 1 & 0.2 \\
\hline Urban & Sedentary & 1 & 1 & 1 & 0.8 & 0 & 0.7 \\
\hline Wetlands & Sedentary & 0 & 3.1 & 1 & 2.4 & 1 & 2.1 \\
\hline
\end{tabular}

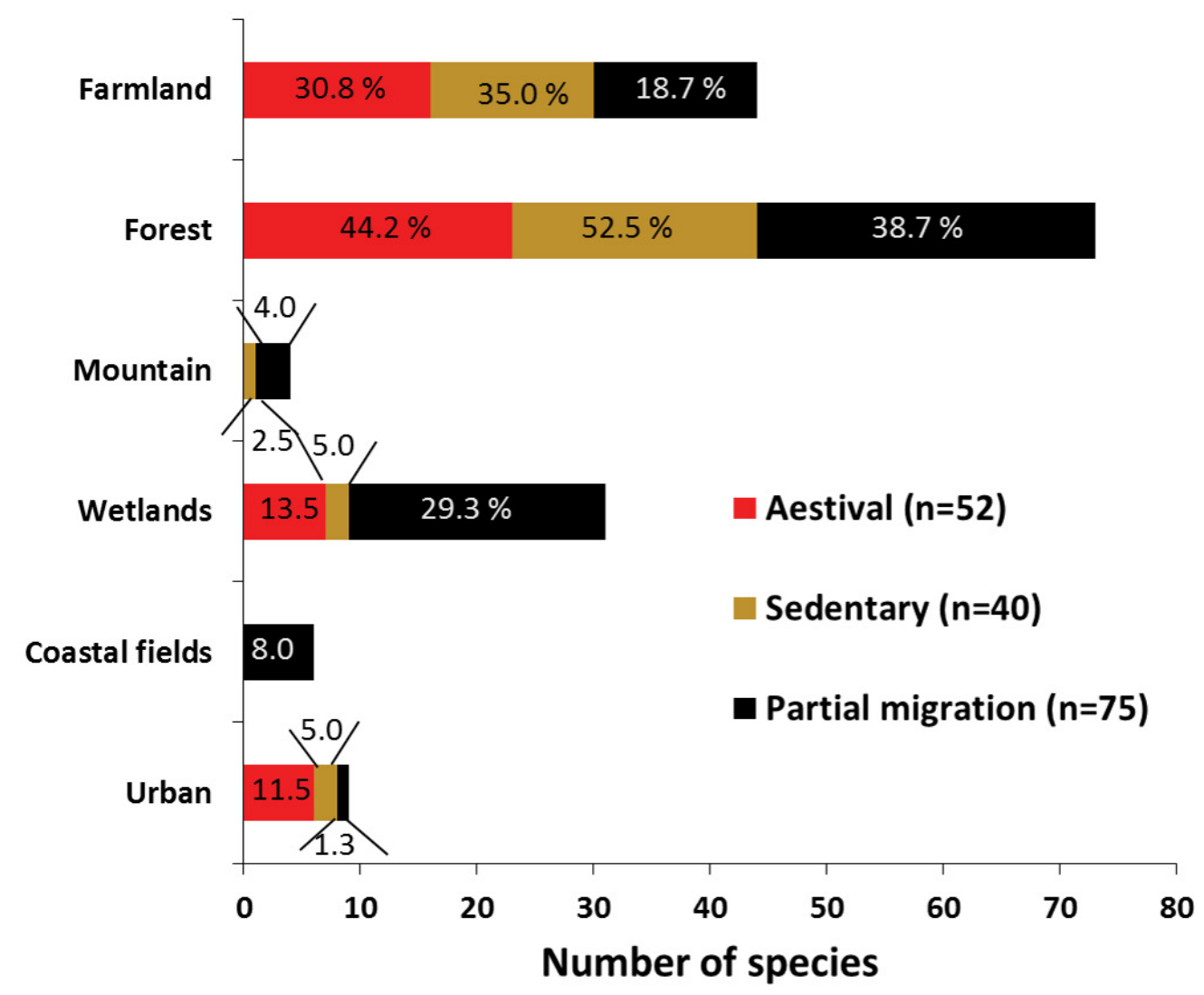

Figure 2: Number of species for each habitat and movement type. Note that the percentages refer to the variable in the legend, not the variable displayed on the $\mathrm{Y}$-axis. 
between variables we did not found any significance.

Contingency tables have been also developed to confirm that we have at least a two-way interaction between these groups of variables and obtain a deeper view of the interaction between variables.

\section{Contingency table two-dimension analysis}

Taking the variables studied for pairs, the following have come out statistically significant:

Habitats X movements: The "Habitats - Movements" statistical analysis showed a high statistical significance $(p=$ 0.0004). In a random distribution of data, the percentage of different species movements should be similar in each habitat. The number of species for each habitat and movements,

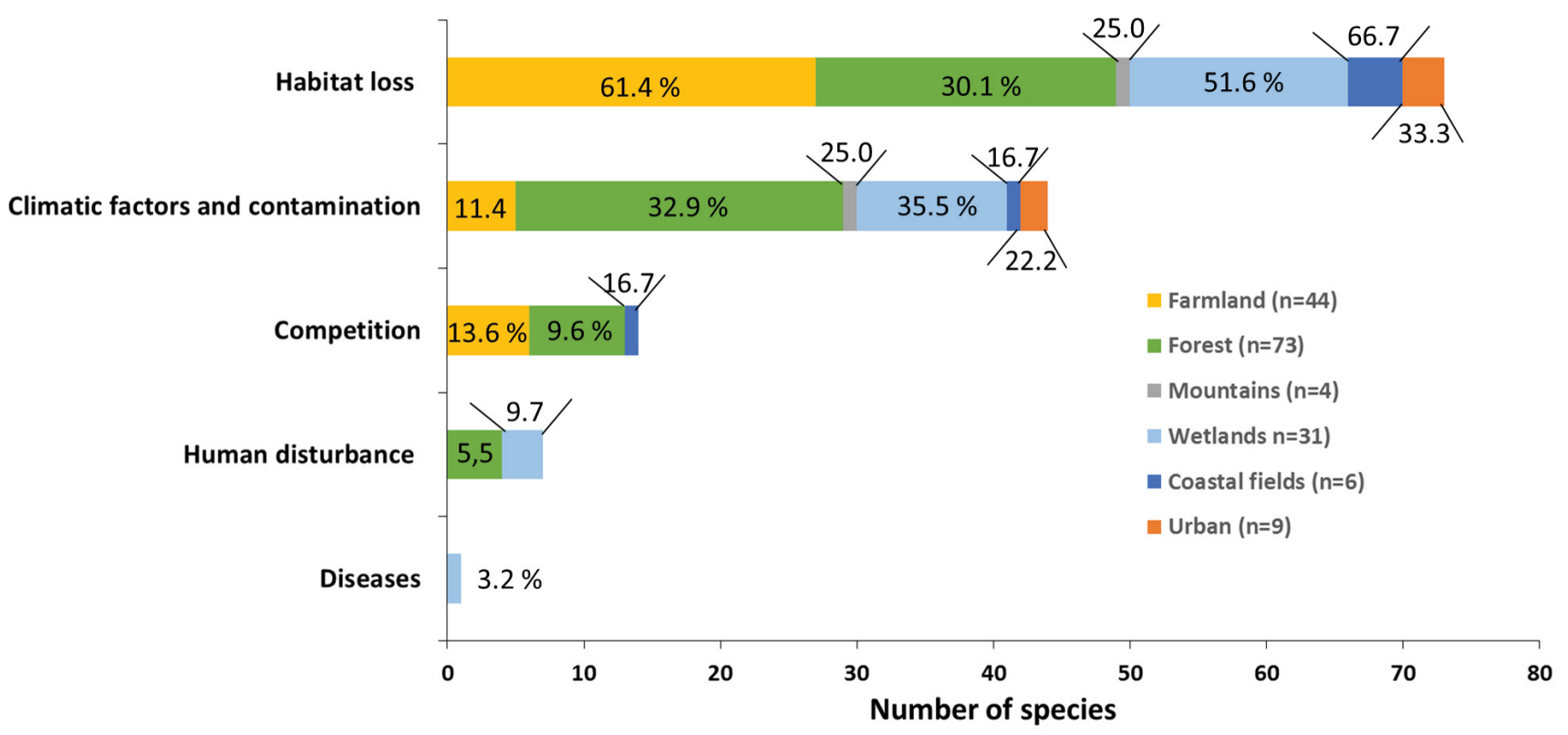

Figure 3: Number of species for each habitat and threat type (the percentages presented include the category "without any known threat"). Note that the percentages refer to the variable in the legend, not the variable displayed on the Y-axis.

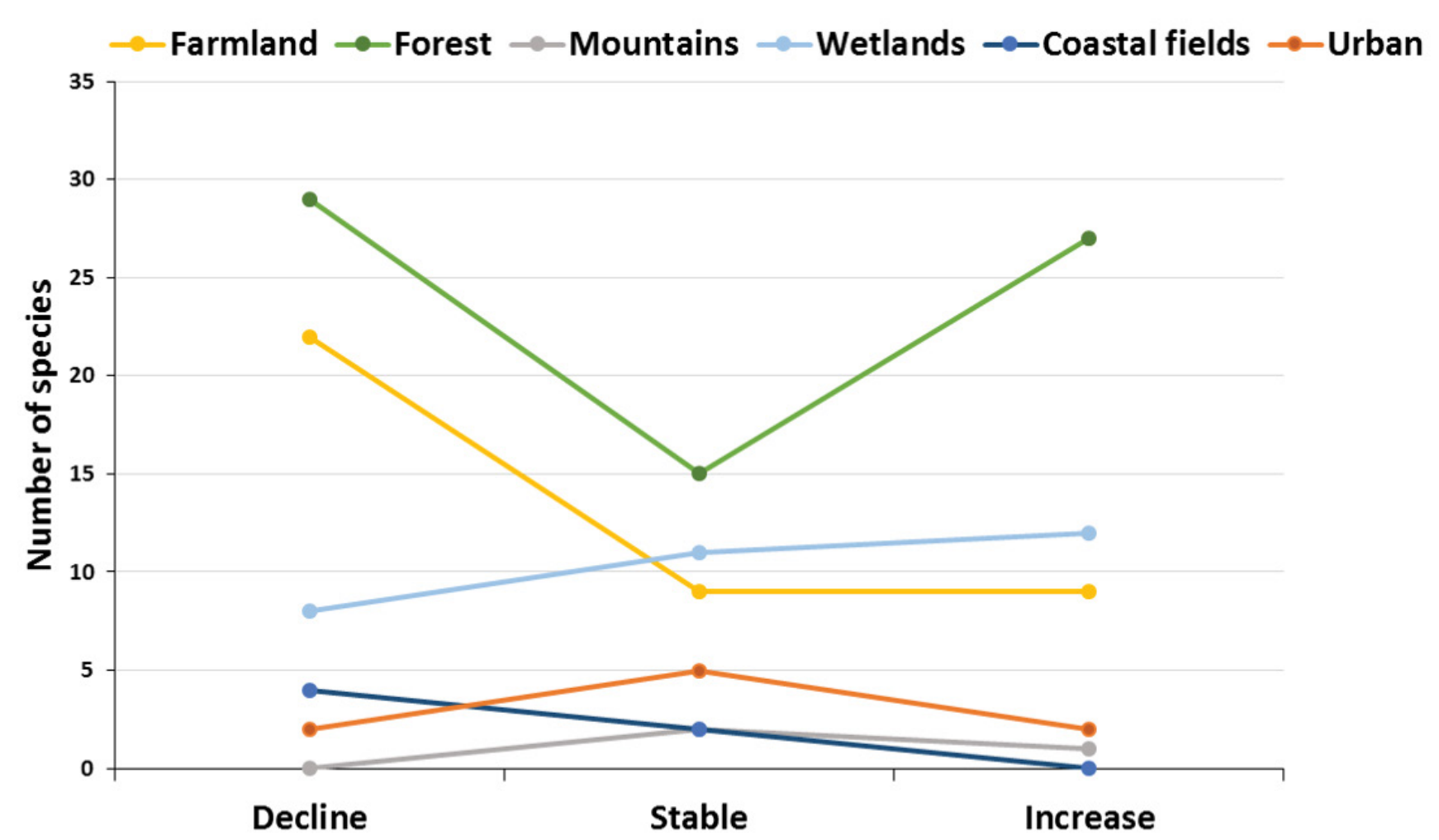

Figure 4: Number of species for each habitat and trend type. 
were graphically compared (Figure 2). Sedentary species are proportionally the most abundant in Farmland and Forest habitats, although forests harbour the majority of all species movements. For the Wetland, Mountain and Coastal field birds, the species with Partial migration are more frequent. The Urban birds have a greater number of Aestival species (Figure 2).

Habitats X threats: The "Habitats - Threats" statistical analysis showed significance $(p=0.03)$. The number of species affected by each threat according to their habitat, were graphically compared (Figure 3 ). In a random distribution of data, the similar percentage of species from different habitats should be affected by each sort of threat. Farmland, Wetland, Coastal field and Urban birds are mainly affected by "Habitat loss". "Climatic factors and Contamination" are more harmful for the Forest birds (Figure 3). Mountain birds are mostly affected by both threats. The other threats weakly affect the different species of each habitat.

Habitats X trend: The "Habitat - Trends" statistical analysis showed a marginal significance $(p=0.07)$. The trends for each group of birds, depending on their habitat, were graphically compared (Figure 4). The Wetland birds have a higher number of species for the "Increase" categories. The Forest birds are polarized between species within Decline and Increase with a few Stable species. The Farmland and Coastal field birds had more species in decline. The Urban and Mountain birds have a higher number of species for the "Stable" category (Figure 4).

Movements X threats: The "Movements - Threats" statistical analysis showed significance $(p=0.02)$. The number of species for each threat and movements, were graphical- ly compared (Figure 5). In a random distribution of data, the similar percentage of different species movements should be affected by each sort of threat. The threat "Habitat loss" is affecting severely species with the different type of Movements. The threat "Climatic factors and Contamination" affects in a lesser grade species with the different type of Movements. The rest of the threats weakly affects species with different type of Movements (Figure 5).

In the rest of the possible two-dimension combination between variables we did not found any significance.

\section{Discussion}

An analysis of the relationship between Habitats, Movements, Threats and Trends for the common European bird species is presented in this work. This study is devoted for common birds, because for the threatened species these factors are much better known. It is a theoretical study that tries to investigate the relationships between the four main variables that condition the state of the populations.

Log linear analyses (of 3 variables) have shown that there is interaction between variables in two different groups of factors. The first one, which involves the variables "Habitats", "Movements" and "Threats", has only marginal significance, but it is showing, looking also the contingency tables analyses with just two variables, that we might be having an homogeneous association, with all the variables being dependent from the rest. We can then conclude that "Threats" depends on both "Habitats" and "Movements" of common birds, which also interact between them as it is observed in the contingency table analyses.

On the other hand, the combination of variables "Habi-

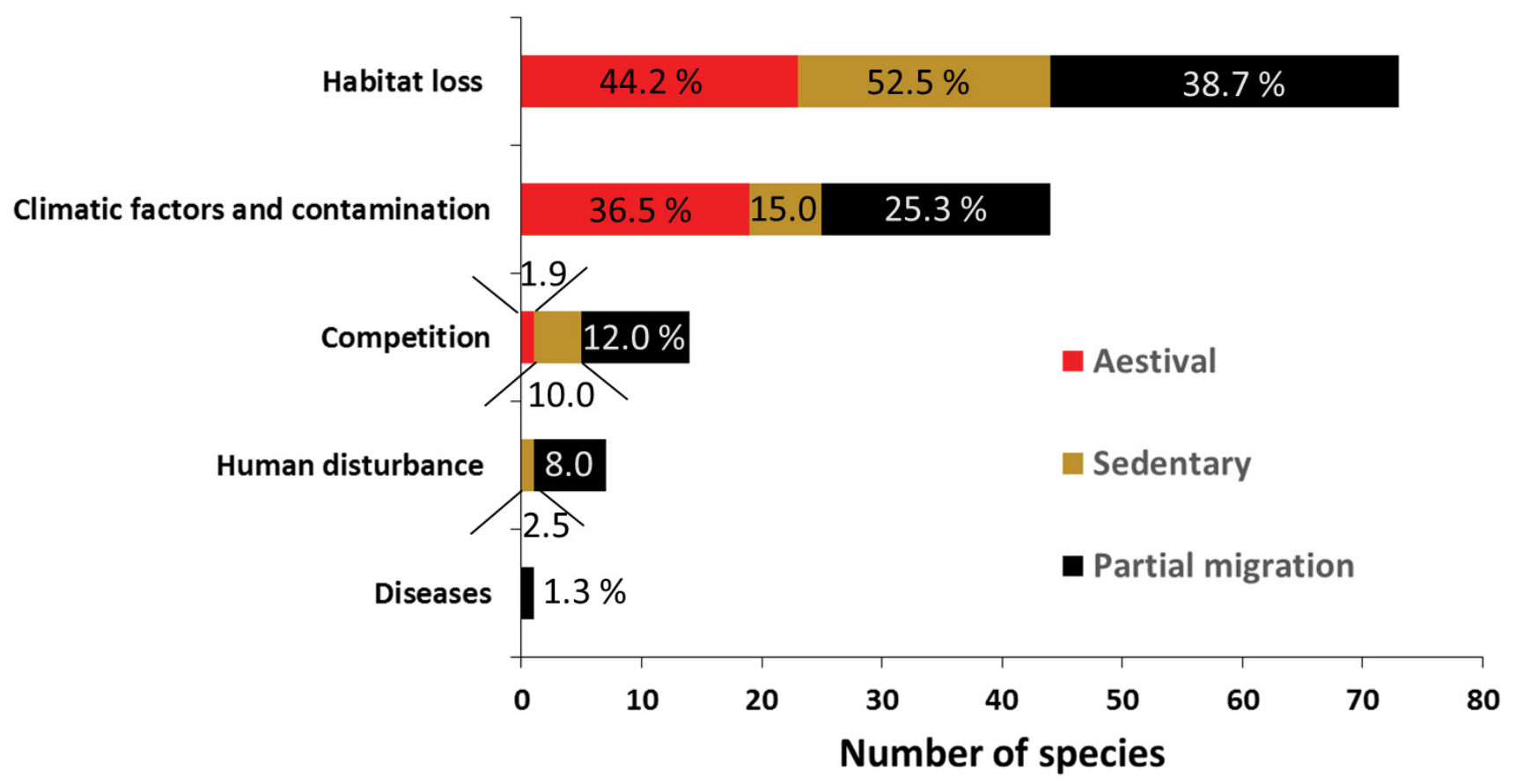

Figure 5: Number of species for each movement and threat type (the percentages presented include the category "without any known threat"). Note that the percentages refer to the variable in the legend, not the variable displayed on the $\mathrm{Y}$-axis. 
tats", "Movements" and "Trends", could have a conditional independence between "Movements" and "Trends" that are not dependent between them. In this second case, we can observe in the contingency tables analyses that "Movements" and "Trends" do not have significance between them, contrary to the relation found between "Habitats" and "Movements" (high) and "Habitats" and "Trends" (marginal). These results let us to hypothesize that trends in European common birds seems not affected by the type of movement they perform but these trends are slightly influenced by the habitat they occupy which is at the same time associated with their movements.

The most important threat for common species in Europe is "habitat loss" (Figure 3 and Figure 5), which is also the most significant factor threatening bird species worldwide [16] followed by "Climatic factors and contamination" which especially affects forest birds (Figure 3). As threats are dependent on both habitats and movements, we can extract that Habitat loss will affect severely Farmland Sedentary birds (Figure 3 and Figure 5). In addition, Sedentary birds are the most numerous in Farmland habitat (Figure 2). On the other hand, Climatic factors and Contamination are more harmful Forest Aestival birds (Figure 3 and Figure 5 ) being the Forest habitat the one that harbors more percentage of species and for all movements (Figure 2).

In the global analysis of this work, the threats for Farmland birds already mentioned in previous works stand out [8-11]. Common Farmland birds have declined sharply, especially in the 1980s, a pattern that could be evidenced by statistical analysis from this work (Figure 4). This decline is associated with increased agricultural practices across Europe (intensification), affecting nesting and foraging opportunities $[6,7,10,17]$. Lowland farmland provides habitat for nearly 120 bird species of European Conservation Concern (SPECS) across Europe, the largest number of species with this concern supported by any habitat [17]. Agricultural intensification has had deleterious effects on bird populations at a pan-European scale and should be regarded as a major anthropogenic threat to biodiversity, comparable to global climate change and environmental pollution $[8,11,17]$. The most important changes affecting common farmland birds are hedgerow loss, land drainage, increased mechanisation, increased fertiliser and pesticide use, reduction of spring cultivation, simplification of crop rotations, changes in crop use and loss of farm diversity [5].

Many of these changes were influenced by European Union (EU) policies, such as the Common Agricultural Policy $[4,7]$. There is a related debate on how the Common Agriculture Policy might be modified $[6,7,9,17]$, because the costs of the environmental problems caused by intensive agriculture are as high as the costs of agricultural support [17]. Conservation efforts need to be directed toward financial support for those farming systems compatible with biodiversity conservation, rewarding farmers making environmental improvements to their lands [17].

Populations of common Forest birds in Europe have remained relatively stable over the last decades. The results of this work indicate that the species are distributed between the extremes with species that are increasing (probably the more generalist birds) and others that are in decline (probably more specialists) while very few species maintain a stable trend (Figure 4). In addition, we have noticed that Forest Aestival species are declining more than expected whereas Partial migration ones are declining less than expected (Table $3)$. Biodiversity in western of Europe is under the double negative influence of climate and land use change [18]; generalist birds have tended to prosper, while the specialists have declined, resulting in a more homogeneous environment with lower biodiversity at regional and global scales [9]. Specialised species of both forest and open farmland are declining at a much higher rate than generalists, which are more tolerant to environmental conditions, hence specialisation itself, rather than living in a particular habitat, could be also a good predictor of a species' recent trend [18].

This work has attempted to reduce the lack of basic information on the nature of threats faced by common species, since this gap can reduce the success of conservation efforts [19]. However, not all threats have the same importance and seriousness for the conservation of species in different areas, which can result in having different threats for the same bird species, depending on the location of each population and the specific problems of each place, neither for different species living in the same habitat, as their type of migration could be playing an important role as noted in this work.

The conventional dominant model of conservation tends to work with individual species which are scarce or threatened [16]. Recovery and conservation plans dedicated particularly to a threatened species that is usually emblematic and well known by the population can serve as an "umbrella" for other species. But in this current model of recovery and conservation plans, efforts are not adequately addressed to the threats that affect a large number of species, such as habitat loss [20]. Besides, emblematic species monopolise a large portion of conservation funds, acting as flags at the political level and even influence public opinion, while others, less known, but with similar or greater conservation problems, are relegated to oblivion. In addition, there is a tendency for these emblematic species to become "conservation-dependent", requiring specific management indefinitely over time, correspondingly with unlimited resource consumption [21].

The common species do not have any legal status requiring a "management plan" (recovery or conservation plan); it would be therefore appropriate to work on their conservation through the transversal action plans [22]. It may be important to implement a management model to address the problems that are common for many species, with a particular focus on reducing their non-natural mortality. These "action plans" are transversal and can improve the population status of a wide range of common species that share the same threats and conservation problems, achieving a general improvement of biodiversity.

Plans focused on several species may be more effective than single-species plans because they can take broader viewpoints of threats and be more comprehensive [23], so that the same protection measure can meet the needs of several species, increasing their profitability. In fact, in the 
United States, there is a tendency to prepare such plans [24], but they should take into account that jointly selected species must share similar threats improving biodiversity protection and the allocation of resources [25]. We know now, thanks to the analysis performed in this study that these threats are dependent on movements and habitats they occupy.

In this work with European common birds, we can extract the information that 12 Aestival Forest species are affected by Climatic factors and Contamination or that 9 Sedentary Farmland species are affected by Habitat loss (Table 2) which could guide conservation efforts and provide information about which species could be benefiting from specific measures eradicating certain kind of threats. Threats have been merged in groups in order to make the analyses viable but this could hinder the interpretation of results, so further studies in different places and specific areas are needed to complement the patterns observed.

The action plans dedicated to the prevention of non-natural mortality have the advantage of improving the conservation status of many common species (with few plans, many species sharing the same problems can be helped) whose populations are precisely the ones that are descending to a greater extent [10]. In addition, the number of transversal action plans that would need to be implemented to manage the conservation of these common species is lower than that the conventional system of recovery and conservation plans dedicated to a single species and could increase the efficiency for the solution of a great number of conservation problems, with less expenditure of time and resources.

\section{Acknowledgments}

\section{Financial support and conflict of interest}

The work presented here was carried out without any funding. Authors have not a conflict of interest to declare.

\section{References}

1. Leakey RE, Lewin R (1998) La sexta extinción. Tusquets Editores, Barcelona, Espana.

2. Koh LP, Dunn RR, Sodhi NS, et al. (2004) Species coextinctions and the biodiversity crisis. Science 305: 1632-1634.

3. Thomas JA, Telfer MG, Roy DB, et al. (2004) Comparative losses of British butterflies, birds and plants and the global extinction crisis. Science 303: 1879-1881.

4. De Heer M, Kapos V, Ten Brink BJE (2005) Biodiversity trends in Europe: Development and testing of a species trend indicator for evaluating progress towards the 2010 target. Philos Trans R Soc Lond B Biol Sci 360: 297-308.

5. Gregory RD, Van Strien A, Vorisek $P$, et al. (2005) Developing indicators for European birds. Philos Trans R Soc Lond B Biol Sci 360: 269-288.

6. Gregory RD, Vorisek P, Noble DG, et al. (2008) The generation and use of bird population indicators in Europe. Bird Conservation International 18: S223-S244.
7. Van Strien AJ, Pannekoek J, Gibbons DW (2001) Indexing European bird population trends using results of national monitoring schemes: A trial of a new method. Bird Study 48: 200-213.

8. Gaston KJ, Fuller RA (2007) Commonness, population depletion and conservation biology. Trends in Ecology \& Evolution 23: 1419.

9. Gregory RD, Van Strien A (2010) Wild bird indicators: Using composite population trends of birds as measures of environmental health. Ornithological Science 9: 3-22.

10. Inger R, Gregory R, Duffy JP, et al. (2014) Common European birds are declining rapidly while less abundant species' numbers are rising. Ecology Letters 18: 28-36.

11. Donald PF, Green RE, Heath MF (2001) Agricultural intensification and the collapse of Europe's farmland bird populations. Proc Biol Sci 268: 25-29.

12. Nicolai J, Singer D, Wothe K (1994) Birds of Britain \& Europe. Collins Nature Guides.

13. Peterson R, Mountfort G, Hollom P (1991) Guía de campo de las aves de España y de Europa. Ediciones Omega, Barcelona.

14. Hagemeijer WJ, Blair MJ (1997) The EBCC atlas of European breeding birds. Poyser, London.

15. R Core Team (2014) R: A language and environment for statistical computing. R Foundation for Statistical Computing, Vienna, Austria.

16. Sodhi NS, Ehrlich PR (2010) Conservation biology for all. Oxford University Press, Oxford, Reino Unido.

17. Donald PF, Pisano G, Rayment MD, et al. (2002) The common agricultural policy, EU enlargement and the conservation of Europe's farmland birds. Agriculture, Ecosystems \& Environment 89: 167-182.

18. Julliard R, Jiguet $F$, Couvet D (2003) Common birds facing global changes: What makes a species at risk? Global Change Biology 10: 148-154.

19. Lawler JJ, Campbell SP, Guerry AD, et al. (2002) The scope and treatment of threats in endangered species recovery plans. Ecological Applications 12: 663-667.

20. Campbell SP, Clark JA, Crampton LH, et al. (2002) An assessment of monitoring efforts in endangered species recovery plans. Ecological Applications 12: 674-681.

21. Scott JM, Goble DD, Haines AM, et al. (2010) Conservation-reliant species and the future of conservation. Conservation Letters 3: 91-97.

22. Balmori A (2015) Eficacia de los Planes de Recuperación y Conservación de las aves amenazadas en España: Avanzando en un modelo transversal de conservación y gestión de la fauna amenazada. Ecosistemas 24: 61-77.

23. Boersma PD, Kareiva P, Fagan WF, et al. (2001) How good are endangered species recovery plans? The effectiveness of recovery plans for endangered species can be improved through incorporation of dynamic, explicit science in the recovery process, such as strongly linking species' biology to recovery criteria. BioScience 51: 643-649.

24. Bottrill MC, Walsh JC, Watson JE, et al. (2011) Does recovery planning improve the status of threatened species? Biological Conservation 144: 1595-1601.

25. Clark JA, Harvey E (2002) Assessing multi-species recovery plans under the endangered species act. Ecological Applications 12: 655-662.

DOI: $10.36959 / 742 / 229$

Copyright: (C) 2020 Balmori A, et al. This is an open-access article distributed under the terms of the Creative Commons Attribution License, which permits unrestricted use, distribution, and reproduction in any medium, provided the original author and source are credited. 


\section{Annex 1: Criteria for inclusion in each threat category}

\section{Climatic factors and Contamination}

"Climatic factors" refers to climatic aspects regarding adverse climatological circumstances such as torrential rains, droughts in sub-Saharan areas (affecting migratory birds), storms, extremely hard winters (which increase the mortality of small birds), as well as global warming caused by climate change. "Contamination" includes a wide variety of aspects such as air pollution, problems with phytosanitary treatments, insecticides and herbicides in farmland and forestry work (fertilisers, nitrates, armored seeds etc.) and water contamination. The problem of Polychlorinated byphenils (PCBs) has also been included, as well as eutrophication and contamination with organic matter. Coastal oil spills and petroleum pollution include discharges of hydrocarbons into the sea and industrial and domestic discharges into rivers. This threat also includes light pollution.

\section{Competition (including predation and lack of food)}

"Competition" is considered to be exercised by other species, either with an alimentary nature or by space. "Predation" refers especially to predation caused by domesticated or feral mammals (dogs, cats, etc.) either on the eggs or on the birds themselves. In cases where predation is caused by invasive species, it has been included in the threat "invasive species". "Lack of food" refers to the reduction of prey populations, the lowest availability of carrion for necrophagous birds in the field, the effects caused by insecticides to insectivorous birds and by herbicides in granivorous birds and the reduction of fish populations for the effects of overfishing on piscivorous birds.

\section{Human disturbance (including fishing, lead poisoning, poisoning, powerlines, run over, spoliation of nests, trapping and hunting, windfarms accident)}

"Human disturbance" refers to disturbances caused by hiking, climbing, nature photography, bathers, river and reservoir navigation, military manoeuvres, vehicular traffic (opening of runways and firebreaks, uncorking, pruning, restocking, clearing of the forest), the construction phase of infrastructure and, in general, disturbances of anthropic origin, irrespective of the fact that they can also cause habitat deterioration, which has been considered a specific threat above. In addition, some farmland activities which coincide with the nesting of several species (for example harvesting coinciding with the breeding of harriers) are included. The threat "fishing" refers to the mortality caused by fishing gear on seabirds and river birds. The consequence of this activity on the decline of prey fish populations is included in the threat "lack of food". "Lead poisoning" differs from "contamination" due to its specific characteristics and affects a wide spectrum of raptors, carrions and aquatic birds. "Poisoning" refers to the illegal use of poisoned baits. On numerous occasions, it is mainly directed to the control of opportunistic predators or feral dogs (especially for hunting or agronomic reasons), but directly or collaterally causes the poisoning of numerous raptors and other necrophagous species. "Powerlines" includes both the electrocution problems in the pylons and those of collision with the aerial cables that affect the species to different degree, in relation to their size and behaviour. "Run over" refers to death on highways and traffic casualties. "Spoliation of nests" includes the capture of eggs or chickens from a nest for possessional or illegal trade. "Trapping and hunting" refers to death by hunting activities, whether by confusion or intentionally. It includes the capture of small birds, which is frequent in Mediterranean countries. "Wind farm accident" refers to deaths caused by wind turbines.

\section{Habitat loss (including forest fires)}

"Habitat loss" is caused by the construction of infrastructures, such as highways, high-speed trains. It also includes urbanisation of natural lands, inadequate forest management, habitat fragmentation, logging with the disappearance of mature forests, changes in farmland landscapes, farmland intensification, destruction of hedges, loss of traditional fallow, increase of irrigation, intensification of vineyards, increase of olive groves to the detriment of herbaceous crops under plastic and afforestation in old farmland or livestock lands. This threat also includes the effects of wetland and grassland overgrazing on vegetation, turbidity of water, soil compaction, trophic competition, the loss of traditional uses, the abandonment of crops (such as mountain crops), loss of trees, open pit mines, the destruction of dunes and the abandonment of salt flats, wetland drainage etc. "Forest fires" considers the effects of forest fires on species.

\section{Diseases (including genetic problems)}

The threat "Diseases" affects species sustainability to avian influenza (strain H5N1) which may be threatened by future outbreaks or species which are sensitive to other bird diseases such as duck virus enteritis. "Genetic problems" are defined by genetic hybridization by introduction of other species or varieties. Problems of endogamy or bottlenecks due to population decline, founder effects, etc.

\section{Invasive species}

"Invasive species" includes all the problems created by the introduction of foreign species.

\section{Without any known threat}

"Without any known threat" refers to species for which threats are unknown. 